\title{
Utilization of the current national antimalarial treatment guidelines among doctors in army hospitals in Lagos, Nigeria
}

\author{
Nkechinyere E. Harrison $^{1^{*}}$, Tolulope F. Olufunlayo ${ }^{2}$, Chimere O. Agomo ${ }^{3}$ \\ ${ }^{1}$ Department of Medicine, 68 Nigerian Army Reference Hospital, Yaba, Nigeria; \\ *Corresponding Author: nke1207@yahoo.com \\ ${ }^{2}$ Department of Community Health and Primary Care, College of Medicine, University of Lagos, Idi-Araba, Nigeria \\ ${ }^{3}$ Malaria Research Laboratory, Department of Biochemistry, Nigerian Institute of Medical Research, Yaba, Nigeria
}

Received 16 March 2012; revised 16 April 2012; accepted 28 May 2012

\section{ABSTRACT}

Introduction: Human malaria infection remains a problem of public health importance in many regions of the world. The infection continues to spread particularly in sub-Saharan Africa. In Nigeria, malaria and its treatment have been affected by factors like wrong diagnosis, wrong attitude of the people, unavailability of drugs, fake and substandard drugs, attitude and practices of medical and paramedical personnel. Nigeria's national standard for malaria treatment was recently changed to artemisinin combination therapy. Methods: A descriptive study using a structured questionnaire was administered to all doctors (123) practicing in the three Army hospitals in Lagos. The study was done between March, 2009 and April, 2009. The findings were pre-coded; data entry and analysis was done using EPI INFO version 3.5.1 statistical software. Results: Presumptive/clinical diagnosis was still a common practice for diagnosing malaria among doctors, as well as the use of microscopy. None of the doctors had the facility for diagnosing malaria with rapid diagnostic test in their hospitals. Fifty one percent of the doctors stated that they utilized the current National antimalarial treatment guidelines. Significant proportion of the doctors used Artemisinin-based Combination Therapy (ACTs) as first line treatment of uncomplicated malaria in adults and children. Chloroquine was the commonest drug for first line treatment of uncomplicated malaria in pregnancy. Only $45.5 \%$ of the doctors had correct knowledge of Intermittent Preventive Treatment in pregnancy (IPTp), while $33.3 \%$ knew the stage of pregnancy in which a pregnant woman should receive IPTp with sulphadoxine-pyrimethamine.
Awareness and the content of knowledge of the current National antimalarial treatment guideline were not affected by the category or current position of the doctors. Thirteen percent of the respondents had attended training/update workshop organized by the Federal Ministry of Health (FMOH) Roll Back Malaria programme on the current National antimalarial treatment guideline. Conclusion: The study showed that there is need to improve use of recommended antimalarial medicines for all categories of patients by doctors in Army Hospitals in Lagos. More effort should be made by the Federal Ministry of Health and other stakeholders to organize training and refresher courses on the current National antimalarial treatment guidelines for all categories of health care providers.

Keywords: National Antimalarial Treatment Guidelines; Antimalarial Treatment; Army Hospitals

\section{INTRODUCTION}

Human malaria infection remains a problem of public health importance in many regions of the world. The malaria infection continues to spread particularly in subSaharan Africa due to a combination of factors: weak health systems, wrong attitude of the people, large population movement, deteriorating sanitation, climatic changes, spreading drug resistance and in certain cases uncontrolled development activities [1-4].

Nigeria accounts for a quarter of all malaria cases in the World Health Organization (WHO) African region [5]. Malaria reduces $1 \%$ of Nigeria's Gross National Product (GNP) annually and 25\% of household income is expended on malaria control and treatment [6].

As a response to the antimalarial drug resistance situa- 
tion, WHO now recommends that treatment policies for falciparum malaria in all countries experiencing resistance to monotherapies such as chloroquine, sulphadoxine-pyrimethamine and amodiaquine, should be combination therapies; preferably those containing an artemisinin derivative [7]. Nigeria made this paradigm shift to use artemisinin-based combination therapy (ACT) as first line and second line treatment of uncomplicated malaria in 2005 [8].

The policy and guidelines recommend the use of ACTs for the treatment of uncomplicated malaria, with artemether-lumefantrine (AL) being the first choice. Parenteral quinine or parenteral artemisinin derivatives are recommended for the treatment of severe malaria, quinine for the treatment of malaria in all trimesters of pregnancy while ACTs can be used for treatment of malaria in the second and third trimesters of pregnancy. It also recommends the use of intermittent preventive therapy in pregnancy (IPTp) using sulphadoxine-pyrimethamine (SP) [8,9].

This study assessed the antimalarial treatment practices among doctors in Army Hospitals in Lagos, Nigeria vis a vis the current national antimalarial treatment guidelines.

\section{METHODS}

Lagos lies in south western Nigeria, in the gulf of Guinea west of the Niger River delta. It is located on longitude $3^{\circ} 24^{\prime} \mathrm{E}$ and latitude $6^{\circ} 27^{\prime} \mathrm{N}$. The monthly rainfall varies from as low as $35 \mathrm{~mm}$ (1.5 inches) in January to as high as $300 \mathrm{~mm}$ (12 inches) in July [10].

This was a descriptive cross-sectional study. All doctors (123) working in the three existing Army Hospitals in Lagos were included in the study. The three hospitals are 68 Nigerian Army Reference Hospital Yaba, Military Hospital Ikoyi and 81 Division Hospital Obalende.

The data was collected over 6 weeks, from March to April 2009. One hundred and twenty three self-administered, structured questionnaires were distributed and all were returned properly filled, thus the response rate was 100\%. The Nigeria National Antimalarial Treatment Policy and Guidelines 2005 were used in this study as benchmark for recommended malaria treatment practices $[8,9]$.

This study was reviewed and approved by the Ethical Committee of the 68 Nigerian Army Reference Hospital Yaba, Lagos, one of the three army hospitals in Lagos. Informed consent was obtained verbally from each respondent prior to filling the questionnaire. Data entry and processing was done using Epi-Info version 3.5.1 (CDC, Atlanta USA). Test of significance was done using chisquare test, and statistical significance was considered at 95\% confidence level.

\section{RESULTS}

The majority of the doctors (41.5\%) were between 26 30 years of age with a mean age of $(36.3 \pm 10.5)$ years. Less than half of the respondents (45.5\%) were medical officers, while slightly over half (55.3\%) had practiced medicine for less than five years. Only $13 \%$ of the doctors were army personnel, the rest being civilians in employment at the hospital (Table 1).

Seventy three (59.3\%) of the doctors were aware of the current national antimalarial treatment guidelines, while $51.2 \%$ claimed they utilized the guidelines in managing malaria cases.

None of the doctors had facility for diagnosing malaria with rapid diagnostic tests in their hospital. Presumptive/ clinical diagnosis (clinical suspicion) of malaria was practiced by the majority of doctors (85.4\%) in Army hospitals in Lagos, and a similar proportion stated that they also diagnosed malaria with the aid of microscopy (85.4\%). Only 16 doctors (13\%) had attended any update workshop/training on the current national antimalarial treatment guidelines; and possessed a copy of the current national antimalarial treatment guidelines.

ACTs were the commonest drugs reported by respondents as being used for first line treatment of uncomplicated malaria in adults and children (77.2\% and 65.8\% respectively). The recommended first line ACT (artemether-lumfantrine) was selected as first drug of choice

Table 1. Sociodemographic profile of respondents $(\mathrm{N}=123)$.

\begin{tabular}{|c|c|c|}
\hline Variable & Frequency & $\%$ \\
\hline \multicolumn{3}{|l|}{ Age (years) } \\
\hline $21-25$ & 6 & 4.9 \\
\hline $26-30$ & 51 & 41.5 \\
\hline $31-35$ & 17 & 13.8 \\
\hline $36-40$ & 11 & 8.9 \\
\hline$>40$ & 38 & 30.9 \\
\hline \multicolumn{3}{|l|}{ Years of Practice } \\
\hline$<5$ & 68 & 55.3 \\
\hline $5-9$ & 15 & 12.2 \\
\hline $10-14$ & 5 & 4.1 \\
\hline $15-19$ & 3 & 2.4 \\
\hline$>20$ & 32 & 26.0 \\
\hline \multicolumn{3}{|l|}{ Current Rank } \\
\hline Consultant & 26 & 21.1 \\
\hline Medical Officer & 56 & 45.5 \\
\hline House Officer & 41 & 33.3 \\
\hline
\end{tabular}


by $46.6 \%, 42.3 \%$ and $2.4 \%$ of doctors for treating adults, children and pregnant women respectively, while Artemether and SP, combination was indicated as first drug of choice for treating adults, children and pregnant women by $18.7 \%, 10.6 \%$ and $0.8 \%$ of doctors respectively (Table 2). Quinine and artemether were the drugs mostly indicated for treatment of severe malaria (Table 3).

A little less than half (45.5\%) of the doctors had correct knowledge of IPTp, and only one-third knew at which stage of pregnancy a woman should receive IPTp. Concerning their practices of malaria prophylaxis in pregnancy, 33.3\% of respondents indicated the use of SP for their patients/clients, while $26.0 \%$ indicated that they prescribed trimethoprim (daraprim).

Current rank of doctors showed no statistically significant association with awareness of the current national antimalarial treatment guidelines $(\mathrm{P}=0.81)$; also there was no statistically significant association between current rank of doctors and knowledge of IPTp using SP $(\mathrm{P}=0.13)$.

\section{DISCUSSION}

This study revealed a wide variety of antimalarial drug prescription patterns among doctors in Army hospitals in Lagos. Recommended first line ACT was indicated as first drug of choice for adults and children by nearly half of the doctors which the authors consider as an improvement over earlier studies. A Nigerian survey of malaria control practices showed that less than a fifth of the primary and secondary health facilities studied used the recommended malaria treatment guidelines [11]. However it is still of great concern that several years after the introduction of the current malaria guidelines in

Table 2. First line treatment of uncomplicated malaria among doctors in army hospitals in Lagos.

\begin{tabular}{cccc}
\hline Drug & $\begin{array}{c}\text { Adults } \\
\mathrm{n}(\%)\end{array}$ & $\begin{array}{c}\text { Children } \\
\mathrm{n}(\%)\end{array}$ & $\begin{array}{c}\text { Pregnant } \\
\text { women n (\%) }\end{array}$ \\
\hline $\begin{array}{c}\text { ACTs } \\
\text { Chloroquine }\end{array}$ & $95(77.2)$ & $81(65.8)$ & $5(4.1)$ \\
SP $^{2}$ & $3(2.4)$ & $1(0.8)$ & $26(21.1)$ \\
Artemether/artesunate & $5(4.1)$ & $5(4.1)$ & $2(1.6)$ \\
Chloroquine + SP & $3(2.4)$ & $2(1.6)$ & $3(2.4)$ \\
Camoquine + SP & $1(0.8)$ & $2(1.6)$ & $2(1.6)$ \\
Camoquine & $1(0.8)$ & $0(0.0)$ & $13(10.6)$ \\
Quinine & $0(0.0)$ & $0(0.0)$ & $4(3.3)$ \\
Refer/do not treat & $2(1.6)$ & $20(16.3)$ & $40(32.5)$ \\
TOTAL & $123(100.0)$ & $123(100.0)$ & $123(100.0)$ \\
\hline
\end{tabular}

${ }^{1}$ Artemisinin-based Combination Therapy; ${ }^{2}$ Sulphadoxine-pyrimethamine.
Table 3. Drug treatment of severe malaria among doctors in army hospitals in Lagos.

\begin{tabular}{cccc}
\hline Drug & $\begin{array}{c}\text { Adults } \\
\mathrm{n}(\%)\end{array}$ & $\begin{array}{c}\text { Children } \\
\mathrm{n}(\%)\end{array}$ & $\begin{array}{c}\text { Pregnant } \\
\text { women n (\%) }\end{array}$ \\
\hline Quinine & $45(36.6)$ & $50(40.6)$ & $19(15.4)$ \\
Artemether/artesunate & $32(26.0)$ & $20(16.3)$ & $18(14.6)$ \\
Chloroquine & $8(6.5)$ & $9(7.3)$ & $5(4.1)$ \\
Artemether + SP & $4(3.3)$ & $1(0.8)$ & $0(0.0)$ \\
Artemether + CQ & $3(2.4)$ & $4(3.3)$ & $0(0.0)$ \\
Mefloquine + SP & $1(0.8)$ & $0(0.0)$ & $0(0.0)$ \\
Halofantrine & $1(0.8)$ & $0(0.0)$ & $0(0.0)$ \\
Amodiaquine & $0(0.0)$ & $0(0.0)$ & $1(0.8)$ \\
SP & $0(0.0)$ & $0(0.0)$ & $1(0.8)$ \\
Refer & $29(23.6)$ & $39(31.7)$ & $79(64.2)$ \\
Total & $123(100.0)$ & $123(100.0)$ & $123(100.0)$ \\
\hline
\end{tabular}

Nigeria, less than half of doctors in army hospitals indicated that they utilize the recommended ACTs. Factors like clarity of the guidelines, strong evidence and support by opinion leaders especially professional bodies are known to positively influence the use of clinical guidelines [12]. The poor attendance of update/training workshop on current antimalarial treatment guidelines may be partly responsible for the above finding, however further studies evaluating factors affecting utilization of guidelines in Nigeria will be needful.

High level of presumptive diagnosis and treatment of malaria noted in this study could imply over-utilization of ACTs and other antimalarial medicines by these doctors. This is at variance with the World Health Organization (WHO) recommendation on the use of rapid diagnostic tests in malaria diagnosis [13]. Inadequate dosing of antimalarial medicines are known to influence the development of resistant strains of the malaria parasite.

A recent report on malaria in pregnancy showed that the coverage of intermittent preventive treatment (IPTp) of malaria in sub-Saharan Africa is low [14]. This is in keeping with the finding in this study. This could be as a result of poor integration of malaria and reproductive health programs amongst other reasons. However this observation varied with the finding from another study in Benin City, Nigeria where it was noted that $64 \%$ to $83 \%$ of doctors in various cadres had the correct knowledge of what is nationally recommended [15]. This difference could be attributed to the fact that the Benin study focused on doctors already specializing in managing pregnant women who hence are expected to have better knowledge on malaria in pregnancy, but this study cuts across all categories of doctors. 
This study showed a low level of attendance of doctors at training/update workshops on the current national antimalarial treatment guidelines (13\%). Similarly, a low percentage of the doctors (13\%) possessed a copy of the current national antimalarial treatment guidelines, and this is likely to contribute to the less than average compliance observed in this study.

Based on the findings of this study, the authors recommend that more effort should be made by the Federal Ministry of Health and other stakeholders to organize training and refresher courses on the current national antimalarial treatment guidelines. In addition these guidelines should be made available to all health care facilities and individuals through the Nigerian Medical Association and other medical professional associations. Finally, there is need for access to facilities for diagnosing malaria with rapid diagnostic tests in these hospitals.

In conclusion, this study showed that there is need to improve use of recommended antimalarial medicines for all categories of patients by doctors in Army Hospitals in Lagos. More effort should be made by the Federal Ministry of Health and other stakeholders to provide copies of the current National antimalarial treatment guidelines and organize training and refresher courses for all categories of health care providers.

\section{ACKNOWLEDGEMENTS}

We wish to thank Mr. Isa Raji for his assistance in data entry.

\section{REFERENCES}

[1] World Health Organization (2000) Malaria-A worldwide crisis, roll back malaria-promise for progress. WHO, Geneva.

[2] Barat, L.M., Barnet, B.J., Smolinski, M.S., Espey, D.K., Levy, C.E. and Zuker, J.R. (1999) Evaluation of malaria surveillance using retrospective laboratory-Based active case definition in four South Western States, 1995. American Journal of Tropical Medicine and Hygiene, 60, 910914.
[3] Layton, M., Praise, M.E., Campbell, C.C., Advani, R., Sexton, J.D., Bosier, E.M. and Zuker, J.R. (1995) Mosquito-transmitted malaria in New York City, 1993. Lancet, 346, 729-731. doi:10.1016/S0140-6736(95)91503-6

[4] Brook, J.K., Genese, C.A., Bloland, P.B., Zuker, J.R. and Spitalny, K.C. (1994) Malaria probably locally acquired in New Jersey. New England Journal of Medicine, 331, 22-23. doi:10.1056/NEJM199407073310105

[5] World Health Organization (2008) World malaria report. WHO, Geneva.

[6] Federal Ministry of Health (2004) Burden of malaria in Nigeria-An advocacy brochure. FMOH, Abuja.

[7] Medecins Sans Frontieres (2003) ACTNOW to get malaria treatment that works to Africa. MSF Access to Essential Medicines Campaign, Geneva.

[8] Federal Ministry of Health (2005) National antimalarial treatment policy. FMOH, Abuja.

[9] Federal Ministry of Health (2005) National antimalarial treatment guidelines. FMOH, Abuja.

[10] Wikipedia (2012) Lagos. http://www.en.wikipedia.org/wiki/lagos

[11] Federal Ministry of Health (2000) Situational analysis of malaria control in Nigeria. FMOH, Abuja.

[12] Meremikwu, M., Okomo, U., Nwachukwu, C., Oyo-Ita, A., Eke-Njoku, J., Okebe, J., Oyo-Ita, E. and Garner, P. (2007) Antimalarial drug prescription practice in private and publichealth facilities in South-east Nigeria: A descriptive study. Malaria Journal, 6, 1-4.

[13] World Health Organization (2005) Malaria control today: Current WHO recommendations working document. WHO, Geneva.

[14] Hill, J. and Kasembe, P. (2006) Reaching the Abuja target for intermittent preventive treatment of malaria in pregnancy in African women: A review of progress and operational challenges. Tropical Medicine \& International Health, 11, 409-418. doi:10.1111/j.1365-3156.2006.01585.x

[15] Omo-Aghoja, L.O., Aghoja, C.O., Oghagbo, K., OmoAghoja, V.W. and Ezume, C. (2008) Prevention and treatment of malaria in pregnancy in Nigeria: Obstetrician's knowledge of guidelines and policy changes-A call for action. Journal of Chinese Clinical Medicine, 3, 114-120. 